\title{
Evaluation of Fatty Acid Whole Blood Concentrations in the San Cristóbal Galápagos Tortoise (Chelonoidis chathamensis)
}

Khushboo Dass ${ }^{1}$, Gregory A Lewbart ${ }^{\text {Corresp., } 1}$, Juan Pablo Muñoz-Pérez ${ }^{2,3}$, Maryuri I Yépez ${ }^{4}$, Andrea Loyola ${ }^{4}$, Emile Chen ${ }^{5}$, Diego Páez-Rosas ${ }^{3}$

${ }^{1}$ Department of Clinical Sciences, College of Veterinary Medicine, North Carolina State University, Raleigh, North Carolina, United States

2 Faculty of Science and Engineering, University of the Sunshine Coast, Sippy Downs, Queensland, Australia

3 USFQ \& UNC-Chapel Hill Galápagos Science Center (GSC), Universidad San Francisco de Quito, Quito, Ecuador

4 Direcion Parque Nacional Galápagos, Islas Galápagos, Ecuador

59 Oneida Court, Chester Springs, Pennsylvania, Unites States

Corresponding Author: Gregory A Lewbart

Email address: greg_lewbart@ncsu.edu

To continue releasing San Cristóbal Galápagos tortoises housed in managed-care facilities at the Giant Tortoise Breeding Center of Galápagos National Park (Galapaguera de Cerro Colorado) to the Otoy Ecological Farm, health assessments and physical examinations were conducted. As a part of these wellness examinations, blood was drawn from 11 tortoises to analyze fatty acid concentrations. Fatty acid levels can provide insight into the nutritional profiles, immune status, and reproductive health of vertebrates. To the coauthor's knowledge, there is no current information about fatty acids in this species. It was hypothesized that there would be inherent differences based on the different geographic ranges, diets, sex, and age of turtles. It was noted that the $\omega-6 / \omega-3$ ratio was higher for the breeding center than for the ecological farm and that overall polyunsaturated fatty acids (PUFAs) did not have any significant differences. The $\omega-6 / \omega-3$ findings can contribute to a global picture of these fatty acids across taxa, as reptiles are underrepresented in this area of research. Additional results are a resourceful starting point for future investigations into how fatty acids are affected in Galápagos tortoises. 
1 Evaluation of Fatty Acid Whole Blood Concentrations in the San Cristóbal

2 Galápagos Tortoise (Chelonoidis chathamensis)

3

4

Khushboo Dass ${ }^{1}$, Gregory Aldo Lewbart ${ }^{2}$, Juan Pablo Muñoz-Pérez ${ }^{3}$, Maryuri Yépez ${ }^{4}$, Andrea Loyola ${ }^{5}$, Emile Chen ${ }^{6}$, Diego Páez-Rosas ${ }^{7}$

${ }^{1}$ North Carolina State University, College of Veterinary Medicine, Raleigh, North Carolina, United States of America

${ }^{2}$ North Carolina State University, College of Veterinary Medicine, Raleigh, North Carolina, United States of America, greg_lewbart@ncsu.edu

3 Universidad San Francisco de Quito (USFQ) \& UNC-Chapel Hill Galápagos Science Center (GSC) Av. Alsacio Northia, Isla San Cristobal, Galápagos, Ecuador; Faculty of Science and Engineering, University of the Sunshine Coast, QLD, Australia.

4 Dirección Parque Nacional Galápagos, Islas Galápagos, Ecuador

${ }^{5}$ Dirección Parque Nacional Galápagos, Islas Galápagos, Ecuador

69 Oneida Court, Chester Springs, PA 19425, United States of America

7 Universidad San Francisco de Quito, Galapagos Science Center, Islas Galápagos, Ecuador; Dirección Parque Nacional Galápagos, Islas Galápagos, Ecuador

Corresponding Author:

Gregory A. Lewbart

Department of Clinical Sciences, North Carolina State University, College of Veterinary Medicine, Raleigh, North Carolina, United States of America

Email address: kdass@ncsu.edu

\section{Abstract}

To continue releasing San Cristóbal Galápagos tortoises housed in managed-care facilities at the Giant Tortoise Breeding Center of Galápagos National Park (Galapaguera de Cerro Colorado) to the Otoy Ecological Farm, health assessments and physical examinations were conducted. As a part of these wellness examinations, blood was drawn from 11 tortoises to analyze fatty acid concentrations. Fatty acid levels can provide insight into the nutritional profiles, immune status, and reproductive health of vertebrates. To the co-author's knowledge, there is no current information about fatty acids in this species. It was hypothesized that there would be inherent differences 
35 based on the different geographic ranges, diets, sex, and age of turtles. It was noted

36 that the $\omega-6 / \omega-3$ ratio was higher for the breeding center than for the ecological farm

37 and that overall polyunsaturated fatty acids (PUFAs) did not have any significant

38 differences. The $\omega-6 / \omega-3$ findings can contribute to a global picture of these fatty acids

39 across taxa, as reptiles are underrepresented in this area of research. Additional results

40 are a resourceful starting point for future investigations into how fatty acids are affected

41 in Galápagos tortoises.

42 Introduction

43 The San Cristóbal Galápagos tortoise (SCGT), Chelonoidis chathamensis, is endemic

44 to one of the oldest islands in the Galápagos Archipelago (Parent et al., 2008). As the

45 human population in the Galápagos Islands continues to expand, the SCGT faces

46 growing threats and has been categorized as Endangered (EN) in the IUCN red list

47 (Caccone et al. 2017).

49 Recent population genetic analyses have exposed that the population is recovering

50 from a severe bottleneck that depleted its genetic diversity, with a population trend

51 increasing of 2,950 adult individuals (Garrick et al. 2014). The species is protected for

52 any commercial use and international trade under CITES Appendix 1 (Turtle Taxonomy

53 Working Group, 2017). In an effort to restore their population, the SCGT has been

54 brought into managed facilities for care, propagation, and reintroduction into the wild.

55 Maintaining proper husbandry and conducting frequent health screenings at these

56 facilities are essential components to help increase the chance of successful breeding

57 and restocking programs. 
Many health assessments and annual physical examinations include blood collection and analysis for standard hematology and biochemistry parameters (Geffre et al., 2009; Zhang et al., 2011; Gibbons et al., 2013; Lewbart et al., 2018; Carreta et al., 2019). Fatty acid (FA) profiles are not commonly included in health assessments of this or other tortoise species, so reference values have not been established. This study presents the fatty acid profile of $C$. chathamensis from both a captive breeding center and ecological farm where SCGT has been introduced for a conservation and ecotourism initiative and evaluates potential connections to nutritional status, immune function, and reproductive capabilities (Cartland-Shaw et al., 1998, Rustan and Drevon, 2005; Samee et al., 2018).

\section{Materials \& Methods Sampling and Study Area}

For the present study, 16 SCGT were sampled: eleven from the Otoy Ecological Farm (Finca Voluntad de Dios, Cerro Verde, Isla San Cristóbal, Islas Galápagos, Ecuador) and five from Giant Tortoise Breeding Center of Galapagos National Park (La Galapaguera de Cerro Colorado, Isla San Cristóbal, Islas Galápagos, Ecuador). All but one of the SCGT from the Otoy Ecological Farm were male. Alternatively, most of the SCGT from the Breeding Center were juveniles and thus undetermined sex, except for one that was male. All tortoises included in this work were clinically healthy as determined by physical examination, carapace and plastron measurements, blood collection (PCV/TS and biochemistry analysis), temperature, and fecal collection. Samples were collected in the springtime (March, 2018). Typical weather conditions during this time are $25-30^{\circ} \mathrm{C}$ with sunshine and minimal cloud coverage. This study was 
83 performed as part of a population health assessment authorized by the Galapagos

84 National Park Directorate (permit Nos. PC-21-18, PC-57-19) and approved by the

85 Galápagos Science Center and North Carolina State University ethics and animal

86 handling protocol. All handling and sampling procedures were consistent with standard

87 vertebrate protocols and veterinary practices. No experiments were performed and

88 such work is rarely if ever permitted in the Galápagos. Having access to these animals

89 is a privilege and we were taking advantage of a valuable permit related to a health

90 assessment to obtain as much information as possible form these animals while

91 handling and taking blood samples. The data collected are simply a quantification of

92 the fatty acids in this species of Galápagos tortoise.

93

94 Both sampling sites are located on the Northeast side of San Cristóbal Island and share

95 some similarities. The "Galapaguera de Cerro Colorado" is one of a few sanctuaries that

96 breeds Galápagos giant tortoises in captivity. It encompasses 11 hectares (30 acres)

97 and holds 180 free-ranging SCGT that are either in the breeding program or are the

98 offspring of these breeding pairs. The SCGT are allowed to roam freely and have

99 access to a vast quantity of native vegetation, i.e. Manzanillo (Hippomane mancinella).

101 In comparison, the Otoy farm is situated on 16 hectares of private land where SCGT

102 roam freely over an area of 1 and a half hectare with sparsely native plants and

103 introduced trees. The SCGT are fed a mixture of otoy (Otoy yautia) and elephant grass

104 (Pennisetum purpureum) at $5 \mathrm{~kg} /$ tortoise every other day. Additionally, there is a diverse

105 group of non-native fruits at the site including passion fruit (Passiflora edulis), banana 
106 (Musa paradisciaca), guava (Psidium guaiva), plum (Spondias movie) and orange

107 (Citrus aurantium) that the tortoises could have access. A similarity between the diets of

108 both groups is that they have access to opuntia cactus (Opuntia echios).

109

110 Blood Sample Collection and Handling

111 The SCGT chosen to be analyzed as a part of this study were part of an annual

112 continuing health assessment performed as a part of pre-release screening. They were

113 all healthy as determined by physical examination, fecal, and bloodwork parameters.

114 Blood was collected from either the brachial sinus or dorsal coccygeal vein using a

115 heparinized (Heparin Sodium USP, 1000 units/ml; APP Pharmaceuticals, LLC,

116 Schaumburg, IL, USA) $3.0 \mathrm{ml}$ syringe with a 1.0" 22-gauge needle. Two to three drops

117 of whole blood were placed onto a paper card containing anti-oxidant stabilizing agents

118 (PerkinElmer 226 Spot Saver RUO Card, PerkinElmer Health Sciences, Inc., Greenville,

119 SC, USA) and allowed to air dry. Samples on the filter paper were stored at room

120 temperature during transportation. Whole blood was collected and applied to a

121 stabilized Perkin Elmer 206 blood spot card. The card was allowed to dry and then

122 mailed to Lipid Technologies (1600 19 ${ }^{\text {th }}$ Avenue SW, Austin MN 55912) for analysis

123 (Marangoni et al. 2004). Dried blood spots were then punched out into a 16x100mm

124 screw-top test and subjected to direct derivatization with acidic methanol. The resulting

125 fatty acid methyl esters were extracted with hexane and separated by capillary column

126 gas chromatography on a Shimadzu GC2010 gas chromatograph equipped with a $30 \mathrm{~m}$

127 FAMEWAX (Restek, State College, PA) column. Fatty acid methyl esters were 
128 identified by flame ionization detection. Retention times were compared to a mixed fatty

129 acid methyl ester standard from NuChek Prep (Elysian, MN).

130

131 Statistical analysis

132 Differences in fatty acids levels between the $\omega-6 / \omega-3$ ratio and PUFA were

133 analyzed. Sampling sites were determined using a Wilcoxon test to determine if

134 the pairs were statistically the same or not based on current literature. The Holm-

135 Bonferroni correction was applied to set an acceptable p-value cut off, given the

136 high number of comparisons performed and decrease false-positive results. All

137 statistical analysis was performed in R (version 3.4.3, The R Foundation for

138 Statistical Computing).

139

140

\section{Results}

141 The present data shows the median values for the full fatty acid profile from both

142 environments, the Ecological Farm and Breeding Center (Table I). The $\omega-6 / \omega-3$ ratio

143 showed a significant difference $(p$-value $=0.014)$ with the group from the breeding

144 center being higher than the ranch (2.023 vs 1.133, respectively) (Figure I). Comparison

145 of PUFA between the center and the ranch (15.958 vs 13.954 , respectively) was not

146 significantly different $(p$-value $=0.563)($ Figure 2$)$.

147

148 Discussion

149 There is no information regarding normal circulating levels of fatty acids in Galapagos

150 tortoises. Many factors influence the levels of fatty acids including diet, age,

151 physiological status, and stress (Clauss et al., 2007, Joseph et al., 1985; Cartland-Shaw 
152 et al., 1998; Samee et al., 2018), which make it difficult for inter-species extrapolation.

153 These results can provide answers for improving husbandry of animals kept in managed

154 care facilities. Reptiles have unique fatty acids that are important to their biology.

155 Current information in this area has shown a trend of higher $\omega-6 / \omega-3$ ratio in-managed 156 care settings or higher PUFA in wild reptiles (Cartland-Shaw et al., 1998; Clauss et al., 157 2007, Joseph et al., 1985).

158

159 Increasing research in FA effects in human and companion pet nutrition has led to an 160 awareness of some potential adverse effects with dysregulation of $\omega-6 / \omega-3$ ratio.

161 Studies in human nutrition have shown how the shift in the $\omega-6 / \omega-3$ ratio from $1 / 1$ to $5 / 1$ $16250 / 1$ has negative impacts on the function of the neural and cardiovascular system and 163 diseases including cancer and arthritis (Simopolous and Cleland, 2003). One study of 164 companion animals examined FA levels in commercially prepared diets for cats and 165 dogs (Lenox and Bauer, 2013). An increased $\omega-6 / \omega-3$ ratio in cats showed altered platelet functions and improved glucose control via decreased serum insulin. While in 167 dogs, a decreased $\omega-6 / \omega-3$ ratio showed delayed wound epithelialization of wounds, decreased number of neutrophils and leukotrienes B4, decreased CD4+ T lymphocytes, 169 and lower delayed-type hypersensitivity response (Lenox and Bauer, 2013). Our study 170 shows significantly higher values of $\omega-6 / \omega-3$ in the breeding center compared to farm.

171 This could be due to higher levels of $\omega-6$ or lower levels of $\omega-3$ in the diet. While these

172 trends and adverse effects of $\omega-6 / \omega-3$ have been observed in mammals, it is not 173 guaranteed to translate to reptiles. The animals in our study were all healthy and were 
174 not likely in an inflammatory state and thus this was not a confounding factor for the $\omega$ $1756 / \omega-3$.

176

177 Highly unsaturated fatty acids (HUFA) are those that contain multiple double bonds in 178 their carbon chains and therefore are a specific type of PUFA. It has been established 179 that plants and phytoplankton are among the only organisms that can synthesis these 180 PUFAs, making them an essential dietary requirement for most animals, including 181 reptiles (Cartland-Shaw et al., 1998; Rustan and Drevon, 2005). In the present study, 182 there were no significant differences between the two groups for PUFAs, although the 183 breeding center did numerically have higher concentrations than the farm. This finding 184 is in contrast to a study that examines dietary and plasma concentrations of fatty acids 185 between wild and managed tuatara (Sphenodon punctatus) and showed higher plasma 186 concentrations of $\omega-3$ PUFAs of the wild group than the managed group (Cartland187 Shaw et al., 1998). It is interesting to hypothesis why the $\omega-3$ HUFAs of the SCGTs at 188 the breeding center were at such high levels and whether this impacted any of their 189 membrane functionality in terms of fluid balance, immune system, or reproductive 190 capability.

192 Since several tortoises in the present study came from a breeding center, it is essential 193 to discuss the role of FA in reproduction. There have been several reproductive studies 194 conducted to examine the role of fatty acid concentrations on fertility in vertebrates 195 (Cartland-Shaw et al., 1998; Lin et al., 2016; Len Van Tran et al., 2017; Samee et al., 196 2018). In one study using zebrafish, it was concluded that the addition of extra virgin 
197 olive oil extracted from Koroneiki that was rich in oleic acid (18:1 $\omega-9)$ and linoleic acid 198 (18:2 $\omega-6)$ improved the reproductive capability of both males and females in terms of 199 sperm quality (motility, fertility, and hatching), frequency of follicular development, and 200 accelerated hatching (Samee et al. 2018). Another study looked at the importance of $\omega$ 2013 and $\omega-6$ PUFA for male reproductive health in ruminants, including parameters of 202 sperm membrane integrity, maintaining viable sperm during the chilling and freezing 203 process, and testicular development (Len Van Tran et al., 2017). The $\omega-3 / \omega-6$ ratio in 204 boar's was examined and determined to improve testicular development and possibly 205 chances for breeding selection overall libido in animals fed a 1:1 $\omega-3 / \omega-6$ ratio (Yan Lin 206 et al., 2016). While these references are promising developments to be correlated to 207 reproductive success, the SCGT values in the present study were not examined for 208 fecundity. This may be a future avenue to explore once normal FA have been 209 established for this species.

210

211

212

213

214

215

\section{Conclusions}

A unique opportunity to analyze fatty acids vales for healthy Galapagos Tortoises endemic to to San Cristóbal Island was incorporated into the annual health examinations of this population. This novel technique is the first time such a study has been performed in this species. This study supported this finding for the $\omega-6 / \omega-3$ ratio being higher in-managed care animals compared to wild animals. The results did not support the findings of overall PUFAs higher for in-managed care animals. Additionally, parameters influencing the differences of these fatty acids such as diet, sex, and physiologic status, could be examined. 


\section{Acknowledgements}

222 We wish to thank the following people for their support and assistance: Sofía Tacle,

223 Carlos Mena, Stephen Walsh, Philip Page, Kent Passingham, Soledad Sarzosa, Ana

224 Carrión, Sylvia Sotamba and Doug Bibus. Also, we thank the Galapagos National Park

225 Directorate (GNPD) for the permits granted for sampling, and Galapagos Science

226 Center (GSC) for the logistic support during the study. Finally, we thank the GNPD

227 rangers of San Cristobal Island Technical Office, especially Rafael Díaz and Jeffrey

228 Málaga, for their help and assistance in collecting data.

229

230

231

232

233

234

235

236

237

238

239

240

241

242

243

244

245

246

247

248

249

250

\section{References}

Adamovicz L, Bronson E, Barrett K, Deem SL. 2015. Health assessment of free-living eastern box turtles (Terrapene carolina carolina) in and around the Maryland Zoo in Baltimore 1996-2011. J Zoo Wild Med 46 (1): 39-51. https://doiorg.prox.lib.ncsu.edu/10.1638/2014-066R.1.

Caccone A, Cayot LJ, Gibbs JP, Tapia W. 2017. Chelonoidis chathamensis. The IUCN Red List of Threatened Species 2017 doi: http://dx.doi.org/10.2305/IUCN.UK.20173.RLTS.T9019A82688009.en.

Carreta A, Vaden S, Lewbart G, Muñoz-Pérez J, Páez-Rosas D. 2019. Increased BUN and glucose in a group of San Cristobal Galapagos tortoises (Chelonoidis chathamensis). Veterinary Record Case Reports 7: e000699. doi: 10.1136/vetreccr-2018-000699

Cartland-Shaw LK, Cree A, Skeaff CM, Grimmon NM. 1998. Differences in dietary and plasma fatty acids between wild and captive populations of a rare reptile, the tuatara (Sphenodon punctatus) J comp Physiol B 168: 569-580.

Clauss M, Grum C, and Hatt JM. 2007. Fatty acid status of captive wild animals: a review. Zoologische Garten, 76(5-6):382-401. 
252 Garrick, R. C., E. Benavides, M. A. Russello, C. Hyseni, D. L. Edwards, J. P. Gibbs, W. 253 Tapia, C. Ciofi, and A. Caccone. 2014. Lineage fusion in Galápagos giant tortoises. 254 Molecular Ecology 23:5276-5290.

255

256

Joseph JD, Ackman RG, Seaborn GT. 1985. Effect of diet on depot fatty acid composition 257 in the green turtle Chelonia mydas. Comp Biochem Physiol 80B(1): 15-22.

258

Lewbart GA, Griffioen JA, Savo A, Muñoz-Pérez JP, Ortega C, Loyola A, Roberts S, 260 Schaaf G, Steinberg D, Osegueda SB, Levy MG, Páez-Rosas D. 2018. Biochemistry and 261 262 hematology parameters of the San Cristobal Galápagos Tortoise (Chelonoidis chathamensis). Conserv Physiol 6 (1).

263

264

Lenox CE and Bauer JE. 2013. Potential Adverse Effects of Omega-3 Fatty Acids in Dogs 265 and Cats. J V Intern Med. 27:217-226.

266

267

Lin Y, Cheng X, Mao J, Wu D, Sheng-Yu Hu BR, Fang ZF, Che LQ, Wu CM, Li J. 2016.

268

269

270

271

272

273

274

275

276

277

278

279

280

Effects of different dietary n-6/n-3 polyunsaturated fatty acid ratios on boar reproduction. Lipids in Health and Disease 15:31. DOI:10.1186/s12944-016-0193-8

Marangoni F, Colombo C, Galli C. 2004. A method for the direct evaluation of the fatty acid status in a drop of blood from a fingertip in humans: applicability to nutritional and epidemiological studies. Annals of Biochemistry 15;326(2):267-72. doi: 10.1016/j.ab.2003.12.016. PMID: 15003567.

Miller JM, Quinzin MC, Edwards DL, Eaton DAR, Jensen EL, Russello MA, Gibbs JP, Tapia W, Rueda D, Caccone A. 2018. Genome-Wide assessment of diversity and divergence among extant Galápagos giant tortoise species. 2018. Journal of Heredity. 109(6): 611-619. https://doi.org/10.1093/jhered/esy031 
281 Parent CE, Caccone A, Petren K. 2008. Colonization and diversification of Galápagos 282 terrestrial fauna: a phylogenetic and biogeographical synthesis. Phil Trans $R$ Soc B:

283 Biological Sciences. 363(1508):3347-3361. doi: 10.1098/rstb.2008.0118

284

285 Rustan AC and Drevon CA. 2005. Fatty Acid: Structure and Properties. 1-7. doi:

286 10.1038/npg.els.0003894

287

288

Samee SM, Mantieghi N, Estevez A. 2019. Zebrafish as a model to screen the potential 289 fatty acids in reproduction. Zebrafish 16(1): 47-64. https://doi-

290 org.prox.lib.ncsu.edu/10.1089/zeb.2018.1641

291

292

Simopoulos, AP and Cleland LG. 2003. Omega-6/omega-3 essential fatty acid ratio:

293

The scientific evidence. Basel: Karger.

294

295

Tran LV, Malla BA, Kumar S, Tyagi AK. 2017. Polyunsaturated Fatty Acid in Male 296 Ruminant Reproduction - A Review. Asian Australas J Anim Sci. 30(5): 622-637. 297 doi: $\underline{10.5713 / a j a s .15 .1034}$

298

299

300

Turtle Taxonomy Working Group. 2017. Turtles of the World: annotated checklist and 301 atlas of taxonomy, synonymy, distribution, and conservation status. In: Rhodin AGJ, 302 Iverson JB, van Dijck PP, Saummure RA, Buhlmann KA, Pritchard PCH, Mittermeier 303 RA, editors. Conservation biology of freshwater turtles and tortoises: a compilation 304 project: a compilation project of IUCN/SSC tortoise and freshwater turtle specialist 305

306 group. Chelonian Research Monographs 7(8):125- 132. doi: https://doi.org/10.3854/crm.7.checklist.atlas.v8.2017 
Figure 1

Fatty acid $\omega-6 / \omega-3$ ratio in Galapagos Tortoise

Fatty acid concentrations of $\omega-6 / \omega-3$ ratio in San Cristóbal Galapagos Tortoise, Chelonoidis chathamensis

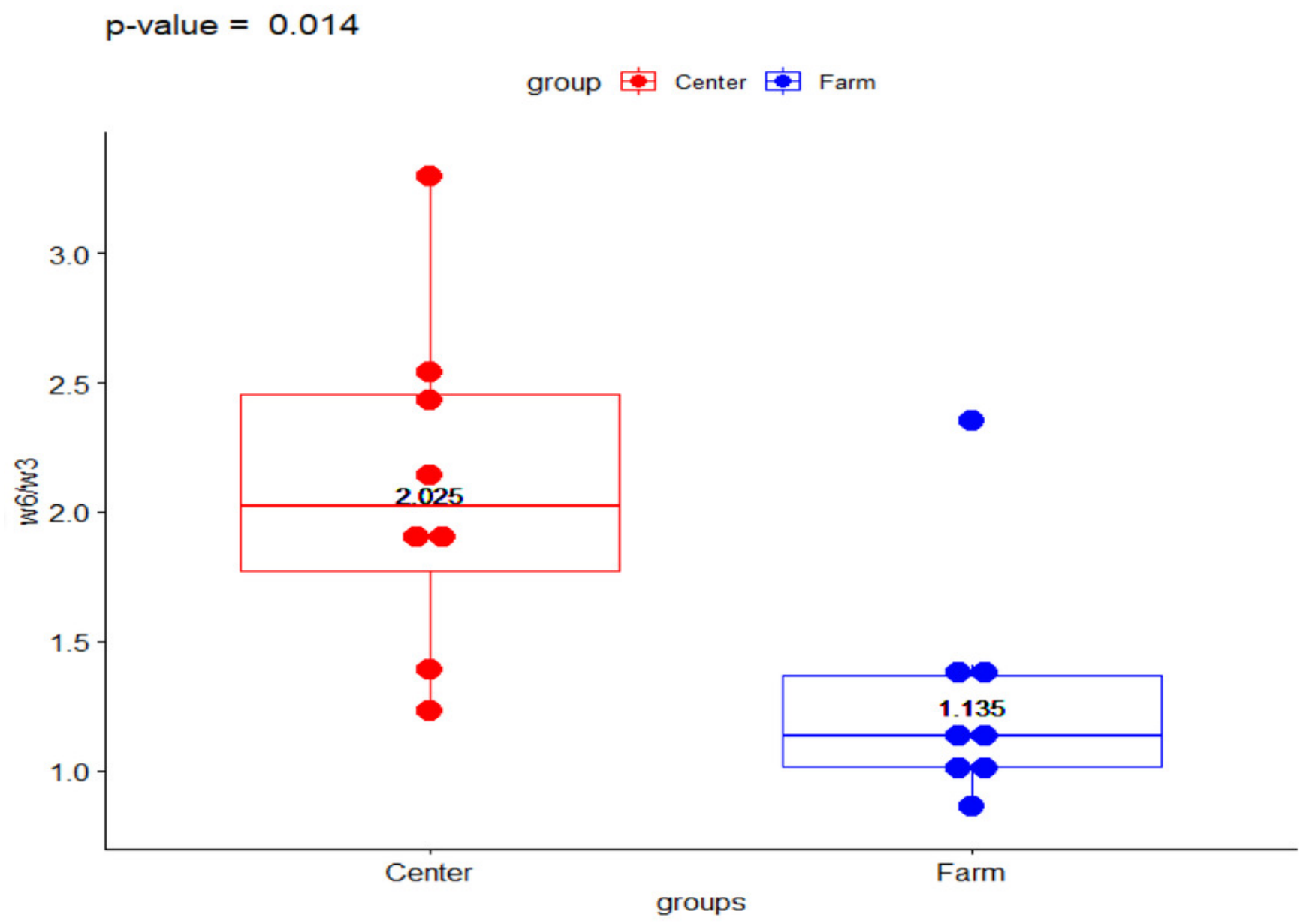


Figure 2

Polyunsaturated fatty acids in Galapagos tortoises

Polyunsaturated fatty acids concentrations in San Cristóbal Galapagos Tortoise, Chelonoidis chathamensis

$p$-value $=0.564$

group $\$$ Center $\$$ Farm

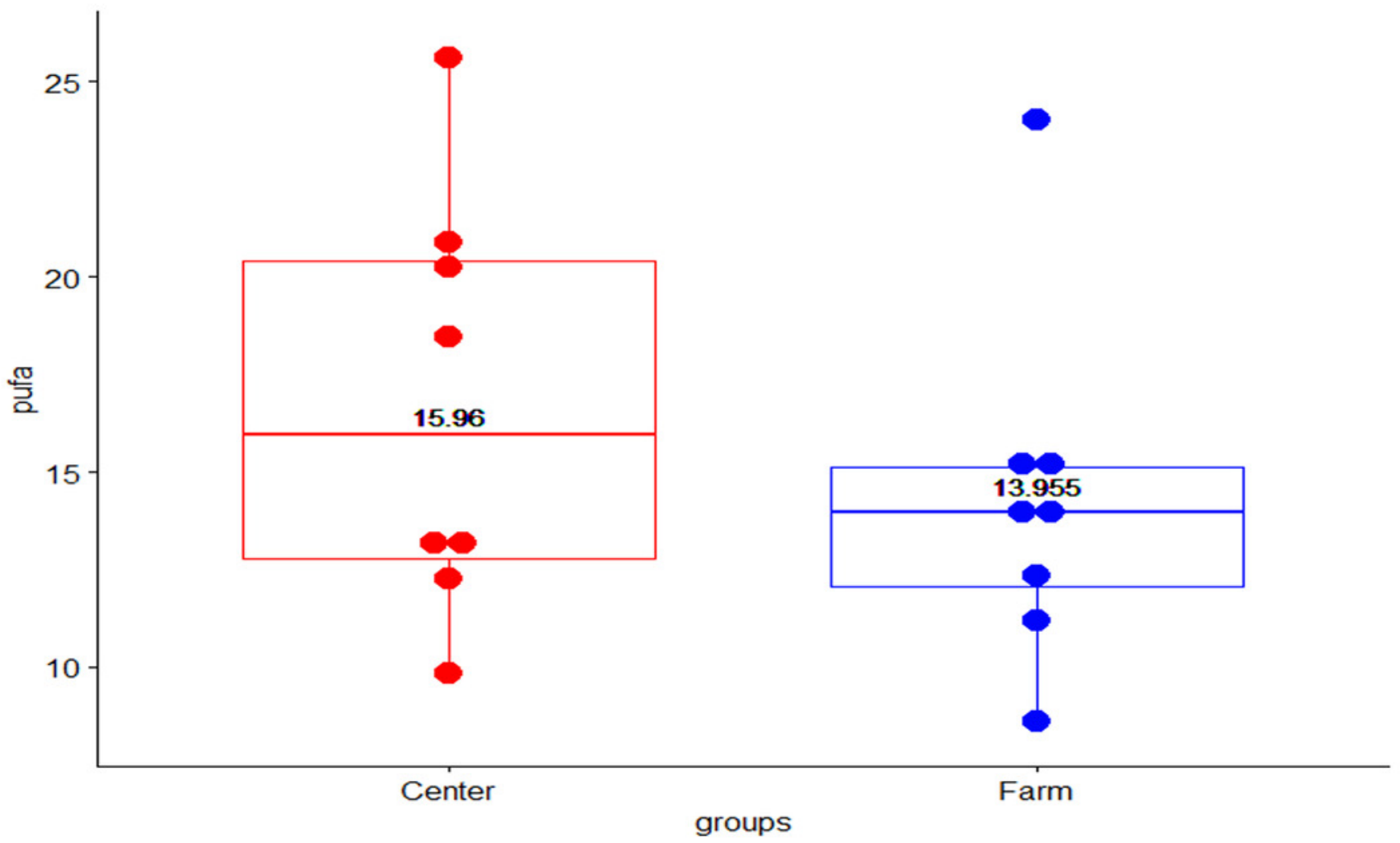




\section{Table $\mathbf{1}$ (on next page)}

Fatty Acid Profile As Percent (\%).

Table I. Total Fatty Acid Profile (Percent, \%) of San Cristóbal Galápagos Tortoises, Chelonoidis chathamensis. 


\begin{tabular}{|c|c|c|c|c|}
\hline Fatty Acid & $\begin{array}{c}\text { Median (Breeding } \\
\text { Center) }\end{array}$ & Mean (Breeding Center) & $\begin{array}{c}\text { Median (Ecological } \\
\text { Farm) }\end{array}$ & $\begin{array}{c}\text { Mean (Ecological } \\
\text { Farm) }\end{array}$ \\
\hline $14: 0$ & 2.16 & $2.10 \pm 0.31$ & 1.72 & $1.86 \pm 0.38$ \\
\hline 14:1 & 1.04 & $1.15 \pm 0.85$ & 1.15 & $1.05 \pm 1.00$ \\
\hline $16: 0$ & 17.35 & $16.96 \pm 1.61$ & 16.38 & $16.52 \pm 1.03$ \\
\hline $16: 1 \omega-7$ & 8.18 & $7.66 \pm 1.07$ & 9.50 & $8.98 \pm 1.84$ \\
\hline $17: 0$ & 0.05 & $0.13 \pm 0.17$ & 0.04 & $0.07 \pm 0.08$ \\
\hline $18: 0$ & 9.03 & $8.95 \pm 1.31$ & 8.36 & $8.72 \pm 1.16$ \\
\hline $18: 1 \omega-9$ & 45.13 & $44.52 \pm 7.68$ & 45.52 & $44.96 \pm 6.37$ \\
\hline $18: 2 \omega-6$ & 3.82 & $4.54 \pm 2.42$ & 3.37 & $4.00 \pm 1.84$ \\
\hline $18: 3 \omega-6$ & 0.06 & $0.10 \pm 0.12$ & 0.06 & $0.08 \pm 0.08$ \\
\hline $18: 3 \omega-3$ & 1.59 & $1.95 \pm 1.05$ & 1.38 & $1.45 \pm 0.87$ \\
\hline $20: 0$ & 0.06 & $0.08 \pm 0.06$ & 0.15 & $0.13 \pm 0.08$ \\
\hline $20: 3 \omega-9$ & 0.23 & $0.23 \pm 0.05$ & 0.26 & $0.26 \pm 0.10$ \\
\hline $20: 3 \omega-6$ & 0.51 & $0.55 \pm 0.23$ & 0.44 & $0.51 \pm 0.16$ \\
\hline $20: 4 \omega-6$ & 2.81 & $3.23 \pm 1.02$ & 2.97 & $4.29 \pm 2.35$ \\
\hline $20: 3 \omega-3$ & 0.04 & $0.04 \pm 0.02$ & 0.02 & $0.06 \pm 0.08$ \\
\hline $20: 4 \omega-3$ & 0.27 & $0.23 \pm 0.10$ & 0.12 & $0.13 \pm 0.12$ \\
\hline $20: 5 \omega-3$ & 2.33 & $2.12 \pm 0.79$ & 1.64 & $1.74 \pm 0.61$ \\
\hline $22: 0$ & 0.18 & $0.20 \pm 0.11$ & 0.24 & $0.21 \pm 0.08$ \\
\hline $22: 1 \omega-9$ & 0.08 & $0.09 \pm 0.02$ & 0.11 & $0.08 \pm 0.06$ \\
\hline $22: 4 \omega-6$ & 0.39 & $0.46 \pm 0.17$ & 0.54 & $0.57 \pm 0.23$ \\
\hline $22: 5 \omega-6$ & 0.13 & $0.14 \pm 0.06$ & 0.08 & $0.09 \pm 0.04$ \\
\hline $22: 5 \omega-3$ & 2.04 & $2.19 \pm 0.82$ & 1.89 & $1.85 \pm 0.41$ \\
\hline
\end{tabular}




\begin{tabular}{|c|c|c|c|c|}
\hline $\mathbf{2 4 : 0}$ & 0.04 & $0.04 \pm 0.04$ & 0.08 & $0.08 \pm 0.07$ \\
\hline $\mathbf{2 2 : 6} \boldsymbol{\omega - 3}$ & 0.13 & $0.16 \pm 0.08$ & 0.15 & $0.21 \pm 0.18$ \\
\hline $\mathbf{2 4 : 1}$ & 0.257 & $0.22 \pm 0.13$ & 0.26 & $0.26 \pm 0.07$ \\
\hline Saturates & 29.68 & $29.37 \pm 3.16$ & 28.56 & $28.32 \pm 1.96$ \\
\hline Monoenes & 47.43 & $46.57 \pm 7.75$ & 46.59 & $47.21 \pm 5.86$ \\
\hline PUFA & 15.05 & $16.01 \pm 5.71$ & 13.44 & $15.28 \pm 4.87$ \\
\hline HUFA & 9.49 & $9.36 \pm 2.44$ & 8.45 & $9.71 \pm 3.33$ \\
\hline T/T & 0.08 & $0.08 \pm 0.03$ & 5.01 & $0.07 \pm 0.03$ \\
\hline Tot. $\boldsymbol{\omega}-3$ & 7.82 & $6.69 \pm 2.23$ & 7.72 & $5.44 \pm 1.57$ \\
\hline Tot. $\boldsymbol{\omega}-6$ & 7.82 & $9.08 \pm 3.76$ & 45.91 & $9.58 \pm 4.22$ \\
\hline Tot. $\boldsymbol{\omega}-\mathbf{9}$ & 47.06 & $45.99 \pm 7.44$ & 1.90 & $46.38 \pm 5.58$ \\
\hline $\boldsymbol{\omega}-6 / \boldsymbol{\omega}-3$ & 1.35 & $1.36 \pm 0.35$ & 2.80 & $1.84 \pm 0.78$ \\
\hline AA/EPA & 1.71 & $1.58 \pm 0.26$ & 39.61 & $2.59 \pm 1.31$ \\
\hline $\boldsymbol{\omega}-3$ HUFA & 49.86 & $50.45 \pm 4.68$ & 60.39 & $43.06 \pm 9.69$ \\
\hline $\boldsymbol{\omega}-6$ HUFA & 50.14 & $49.55 \pm 4.68$ & 1.78 & $59.94 \pm 9.69$ \\
\hline EPA + DHA & 2.44 & $2.28 \pm 0.86$ & & $1.95 \pm 0.65$ \\
\hline
\end{tabular}


